\title{
Three-dimensional adaptive evolution of gravitational waves in numerical relativity
}

\author{
Kimberly C. B. New, ${ }^{1,2}$ Dae-Il Choi, ${ }^{1}$ Joan M. Centrella, ${ }^{1}$ Peter MacNeice, ${ }^{1,3}$ Mijan F. Huq ${ }^{4}$ and Kevin Olson ${ }^{5}$ \\ ${ }^{1}$ Department of Physics, Drexel University, Philadelphia, PA 19104 \\ ${ }^{2} X$-2, MS B-220,Los Alamos National Laboratory, Los Alamos, NM 87545 \\ ${ }^{3}$ Code 931, NASA/GSFC, Greenbelt, MD 20771 \\ ${ }^{4}$ Dept. of Astronomy \& Astrophysics, and Center for Gravitational Physics \& Geometry, \\ The Pennsylvania State University, University Park, PA 16802 \\ ${ }^{5}$ Enrico Fermi Institute, University of Chicago, Chicago, IL 60637
}

\begin{abstract}
Adaptive techniques are crucial for successful numerical modeling of gravitational waves from astrophysical sources such as coalescing compact binaries, since the radiation typically has wavelengths much larger than the scale of the sources. We have carried out an important step toward this goal, the evolution of weak gravitational waves using adaptive mesh refinement in the Einstein equations. The 2-level adaptive simulation is compared with unigrid runs at coarse and fine resolution, and is shown to track closely the features of the fine grid run.
\end{abstract}

04.25.Dm, 04.30.Nk

A new era in general relativistic research is beginning with the inauguration of a worldwide network of gravitational wave observatories including LIGO, VIRGO, GEO, and TAMA [1]. The signals that these instruments will detect are expected to often arise in highly nonlinear, dynamical astrophysical events, such as the final merger of coalescing compact binaries. Fully general relativistic simulations of these events in three-dimensions will be an essential component of the successful detection and interpretation of gravitational wave signals.

A key difficulty in calculating accurate waveforms from these simulations lies in the fact that the models encompass both length and time scales that can vary by an order of magnitude or more. Adequate resolution is needed to model the strong-field dynamics near the sources. Also, the computational domain must be sufficiently large and well-resolved to model the resulting gravitational radiation that, for coalescing compact binaries, can have wavelengths $\sim 10-100$ times the size of the sources. Adaptive techniques that offer dynamic variable resolution are thus crucial for success.

Adaptive mesh refinement (AMR) has undergone significant developments during the past two decades, and remains an active area of research and development. Within the context of general relativistic simulations, Choptuik first implemented the Berger-Oliger [2] AMR method to study critical phenomena in the collapse of massless scalar fields in spherical symmetry [3, 4]; see also [5]. AMR techniques were applied to spherical black hole evolutions in Refs. [6] and [7]. In addition, the construction of initial data for black hole collisions using AMR was studied in Ref. [8], and fixed mesh refinement was employed for a short part of a binary black hole calculation in Ref. [9]. Recently, Papadopoulos, Seidel and Wild [10] carried out 3-D adaptive computation of gravitational waves using a single model equation that describes perturbations of a non-rotating black hole.
We have carried out a 3-D simulation of pure gravitational waves in the Einstein equations using AMR. While our numerical relativity code does solve the full Einstein equations, we have chosen to evolve linearized Teukolsky waves to enable comparison with an analytic solution for this simulation. Overall, our 2-level adaptive simulation successfully reproduces the features of a uniform grid run at the finer resolution.

Our numerical relativity code is based on the ADM code developed by the BBH Alliance [11] and is written in Cartesian coordinates. We have implemented a conformal ADM formalism [12,13], and have incorporated octant symmetry boundary conditions to minimize the number of gridpoints. Parallelism and AMR have been implemented using Paramesh [14], as discussed below. The time evolution is carried out using an iterative Crank-Nicholson method with two iterations [15]. Interpolated Sommerfeld outgoing wave conditions are applied at the outer boundary of the grid [12,13].

Given the complexity of this system of equations and the computational techniques employed, analytic solutions provide an essential testbed for the resulting numerical code. To this end, we use the Teukolsky wave [16], which is a time-dependent solution to the linearized vacuum Einstein equations with geodesic slicing, $\alpha=1$ and $\beta=0$. It is based on a generating function $F(t, r)=$ $\left(A / \omega^{2}\right)(t \pm r) \exp \left(-(t \pm r)^{2} / \omega^{2}\right)$, where $A<<1$ is an amplitude parameter and $\omega$ is the width of the generating function, which is of the same order as the wavelength of the gravitational wave. To set initial data for our simulations, we use a time symmetric, even-parity $L=2, M=0$ Teukolsky wave solution that contains a combination of ingoing and outgoing gravitational waves; see Refs. 16.17]. We set $\omega=1$ and take $A=10^{-6}$, so that the metric perturbation has an initial amplitude $g_{i j}-1 \sim \pm 10^{-5}$. Since $t=0$ is a moment of time symmetry, we have $K_{i j}=0$ initially. 
This data is then evolved in our code with $\alpha=1$ and $\beta=0$. The outgoing waves travel directly toward the edge of the grid. The initially ingoing waves first travel toward the origin, then reflect and move outward. As the overall signal travels away from the origin, it leaves flat space behind. Ref. [16] gives explicit expressions for the metric components $g_{i j}$ that we use as a first-order analytic solution for comparison with our numerical results. All simulations presented in this paper were done with octant symmetry.

We employ an AMR scheme that works on logically Cartesian, or structured, grids. The pioneers in this area are Berger and co-workers [2, 18, 19, who covered the computational domain with structured grids and subgrids that could overlap, take arbitrary shapes, be rotated with respect to the coordinate axes, and be merged with other sub-grids at the same level of refinement. Although this strategy is flexible and memory-efficient, the resulting code can be very complex and difficult to parallelize, especially in 2D and 3D. Simplifications of this basic scheme were developed by Quirk 20] and later by DeZeeuw and Powell [21], who implemented refinement by bisecting the appropriate grid blocks in each coordinate direction and linking the hierarchy of sub-grids as the nodes of a data tree. Wild and Schutz [7,22] have developed a somewhat different approach using hierarchical linked-lists.

Paramesh uses an AMR technique similar to that of DeZeeuw and Powell [21] in which grid blocks are bisected in each coordinate direction when refinement is needed. The grid blocks all have the same logical structure, with $n x b$ zones in the $x$-direction, and similarly for $n y b$ and $n z b$. Thus, in 3D refinement of a block yields 8 child blocks, each having $n x b \times n y b \times n z b$ zones but with zone sizes a factor of two smaller than in the parent block. Refinement can then continue on any or all of these child blocks, with the restriction that the grid spacing is not allowed to change by more than a factor of two, or one refinement level, at any location in the spatial domain. Every grid block has a number of guard cell layers along each of its boundaries, to allow operations between neighboring blocks as well as between different refinement levels. These guard cells are filled with data from neighboring blocks or, if the block is along the edge of the computational domain, with appropriate outer boundary conditions.

Paramesh handles the creation of grid blocks and builds and maintains the data structure (quad-tree in $2 \mathrm{D}$, oct-tree in $3 \mathrm{D}$ ) that tracks the spatial relationships between blocks. It also handles all inter-block communications. In addition, it keeps track of physical boundaries on which particular conditions are to be set, guaranteeing that the child blocks inherit this information from the parent blocks. When executing on a parallel machine, Paramesh distributes the blocks among the available processors to achieve load balance, maximize block locality, and minimize inter-processor communication.

We have implemented our numerical relativity code in the Paramesh framework. For the runs presented in this paper, we use blocks with $n x b=n y b=n z b \equiv n b$ and grid sizes $\Delta x=\Delta y=\Delta z \equiv h$. We also use the same timestep, chosen to give stability on the finest grid, over the whole computational domain. The basic variables are located at the centers of the grid cells. We use secondorder spatial finite differences and a single layer of guard cells. For the AMR runs, we use the restriction (transfer of data from fine to coarse grids) and prolongation (coarse to fine) operators built into Paramesh, which employ linear interpolation. All the calculations presented in this paper were done using a T3E.

A necessary preliminary step in using AMR for numerical simulations is to verify that the code works correctly when run using a single grid with constant $h$ that covers the entire computational domain, i.e. for unigrid simulations. Of particular importance is the convergence behavior of the code. Since we are using finite differences that are second-order accurate in both space and time, we expect that the errors will decrease $\sim \mathcal{O}\left(h^{2}\right)$ as $h \rightarrow 0$.

Using the Teukolsky wave initial data, we ran a set of three runs with increasingly finer grids: $h=0.25\left(16^{3}\right.$ grid), $h=0.125$ ( $32^{3}$ grid), and $h=0.0625$ ( $64^{3}$ grid). In these runs, the outer boundaries of the grid were placed at $x_{\max }=y_{\max }=z_{\max }=4$. Calculation of various error norms demonstrated that, even for these relatively modest resolutions, the code has nearly second-order convergence for the propagation of the wave across the grid. However, as the waves move off the outer edge of the grid, lower amplitude reflected waves travel inward from the outer boundary. When the outer boundary is moved farther from the origin, and thus closer to the asymptotic region in which the Sommerfeld outgoing wave conditions are applicable, the errors due to boundary effects decrease.

To evolve gravitational waves using AMR, we have chosen to use a refinement criterion based on the first derivative of the diagonal components of the conformal metric $\tilde{g}_{i j}=e^{-4 \phi} g_{i j}$, where $e^{4 \phi}=\operatorname{det}\left(g_{i j}\right)^{1 / 3}$. Thus, in each zone we calculate the quantity $\tau=\frac{h}{f}\left(\frac{\partial f}{\partial x}+\frac{\partial f}{\partial y}+\frac{\partial f}{\partial z}\right)$, where $f=\tilde{g}_{i j}-1$ for $i=j$. The computational domain is covered with blocks having $n b^{3}$ physical zones. A block is marked for refinement if $\tau>\tau_{\text {re }}$ for at least one zone in that block, and for derefinement if $\tau<\tau_{\text {de }}$ for all zones in that block, where $\tau_{r e} \geq \tau_{d e}$. Note that both refinement and derefinement in Paramesh are restricted by the requirement that the grid can change by only one refinement level at block boundaries.

We concentrate on 2-level AMR, with level 1 indicating the coarse grid and level 2 the fine grid. For the simulation presented here, we use grid blocks with $n b=4$ and set the outer boundary of the computational domain 
at $x_{\max }=y_{\max }=z_{\max }=7$. After some experimentation, we chose to trigger refinement by $\tau_{r e}=1.75$, and derefinement by $\tau_{d e}=0.875$.

To set up the initial data, we first covered the entire computational domain with level 1 blocks, and used the Teukolsky solution to give $\tilde{g}_{i j}$ and $\phi$. The quantity $\tau$ was then computed and the refinement criterion applied, yielding blocks at level 2. The Teukolsky solution was again applied to these refined blocks to set up $\tilde{g}_{i j}$ and $\phi$. However, when the first derivatives of $\tilde{g}_{i j}$ were taken numerically to compute the conformal connection functions $\tilde{\Gamma}^{i}\left[13\right.$, discontinuities in $\tilde{\Gamma}^{i}$ appeared at the boundaries between level 1 and level 2 blocks. When this data was then evolved forward in time, these initial discontinuities produced errors in other variables. To circumvent these problems, we calculated $\tilde{\Gamma}^{i}$ on a level 2 grid covering the whole domain, and then used the restriction operator to give $\tilde{\Gamma}^{i}$ on the level 1 blocks. This produced a data set without initial discontinuities.

Figure 1 shows the metric component $g_{z z}$ in the equatorial plane for an AMR run with resolution $h=0.25$ on level 1 and $h=0.125$ on level 2 at time $t=5.06$. Initially, only the region near the origin is covered by the fine grid. This refined region moves outward as the wave propagates toward the edge of the grid. As seen in Fig. 1, the region near the origin is derefined as the solution approaches flat space in that region.

It is interesting to compare this AMR solution with unigrid runs using the same computational domain. Figure 2 shows the quantity $g_{z}-1$ along the $x$-axis at $t=5.06$, with the data from the AMR run shown as filled squares. In addition, a short dashed line shows the analytic solution, a solid line the unigrid result with $h=0.125$ ( $56^{3}$ grid, same resolution as the level 2 AMR grid) and a long dashed line the unigrid result with $h=0.25\left(28^{3}\right.$ grid, same resolution as the level $1 \mathrm{AMR}$ grid). Notice that the AMR solution closely tracks the higher resolution unigrid result. When we take the $L_{1}$, $L_{2}$, and $L_{\infty}$ error norms of $g_{z z}$ over the entire computational volume, we find that the AMR results closely track those of the higher resolution unigrid run.

Initially, less than $10 \%$ of the computational volume was covered by the fine grid. This fine grid volume fraction increases as the wave propagates outward, as shown in Fig. 3. Although derefinement in the equatorial plane first occurs around the origin at $t \sim 5$, the total number of fine grid cells continues to increase as the expanding wavefronts propagate outward. The main peak of the wave (located at $x \sim 4.5$ in Fig.2) encounters the outer boundary at $t \sim 7.5$. Around this time, Fig. 3 shows that the fine grid volume fraction begins decreasing as the derefined region behind the wave grows. By $t \sim 9$, most of the Teukolsky wave has left the grid. However, reflected waves traveling inward from the outer boundary cause continued refinement and derefinement, preventing the fine grid volume fraction from dropping to zero.
In this calculation, the savings in computational cost of the AMR run over a unigrid run are modest, even if we neglect the problems with the boundary conditions, since the gravitational wavelength $\lambda$ is comparable to the dimension $L$ of the computational domain. In a more realistic simulation, we would expect $\lambda \ll L$, resulting in significant computational savings. For a full simulation of binary coalescence, one will need to handle multiple scales to evolve both the dynamics of the sources and the propagation of the waves that develop. While the sources are expected to require high resolution, particularly near regions of shocks or shear layers, the waves can likely be handled with lower resolution. Thus, although the resolutions used here are modest compared to those in previous numerical studies of Teukolsky waves, e.g. [23], they are typical of the resolutions likely to used for gravitational waves in full simulations of binary coalescence in the next few years.

While this AMR calculation consitutes an important step toward the ultimate goal of realistic models of gravitational wave sources, more work remains to be done. For example, even though AMR can be used to alleviate the problem of reflected waves by moving the outer boundary even farther from the origin, better outer boundary conditions are still needed [24]. In addition, it will be interesting to test different refinement criteria, including those based on truncation error estimation [4, 10. Finally, we observed that derefinement introduces some high frequency noise, principally in the extrinsic curvature variables. While this did not pose a significant problem for this 2-level run, it can become important when more refinement levels are used. We are experimenting with various ways of removing this noise by using filtering [7] or adding dissipation [25,26], and will report on this work elsewhere.

We are pleased to thank Matt Choptuik for interesting and helpful discussions. This work was supported in part by NSF grant PHY-9722109 at Drexel, and grant number PHY990002P from the Pittsburgh Supercomputing Center (PSC), which is supported by several federal agencies, the Commonwealth of Pennsylvania and private industry.

[1] See, e.g., Gravitational Wave Experiments, eds. E. Coccia, G. Pizzella, and F. Ronga (World Scientific, Singapore, 1995), for articles describing these interferometers.

[2] M. Berger and J. Oliger, J. Computat. Phys. 53, 484 (1984).

[3] M. Choptuik, Phys. Rev. Lett, 70, 9 (1993).

[4] M. Choptuik, in Frontiers of Numerical Relativity, eds. C. Evans, L. Finn, and D. Hobill (Cambridge University Press, Cambridge, England, 1989).

[5] R. Hamade and J. M. Stewart, Class. Quantum Grav. 
13, 497 (1996).

[6] J. Massó, E. Seidel, and P. Walker, in The Seventh Marcel Grossmann Meeting: On Recent Developments in Theoretical and Experimental General Relativity, Gravitation, and Relativistic Field Theories, eds. R. Jantzen, G. Keiser, and R. Ruffini (World Scientific, Singapore, 1996).

[7] L. A. Wild, Ph.D. Thesis (University of Wales, Cardiff, 1996).

[8] P. Diener, N. Jansen, A. Khokhlov, and I. Novikov, grqc/9905079.

[9] B. Brügmann, Int. J. Mod. Phys. D8, 85 (1999).

[10] P. Papadopoulos, E. Seidel, and L. Wild, Phys. Rev. D 58, 084002 (1998).

[11] G. Cook, et al., Phys. Rev. Lett. 80, 2512 (1998).

[12] M. Shibata and T. Nakamura, Phys. Rev. D 52, 5428 (1995).

[13] T. W. Baumgarte and S. L. Shapiro, Phys. Rev. D 59, 024007 (1998).

[14] P. MacNeice, K. M. Olson, C. Mobarry, R. deFainchtein, and C. Packer, Computer Physics Comm., in press 1999 (NASA Contractor report, number NASA/CR1999-209483).

[15] S. A. Teukolsky, gr-qc/9909026 (1999).

[16] S. A. Teukolsky, Phys. Rev. D 26, 745 (1982).

[17] K. Eppley, in Sources of Gravitational Radiation, ed. L. Smarr, (Cambridge University Press, 1979).

[18] M. Berger, Ph.D. Thesis, Stanford University (1982).

[19] M. Berger and P. Colella, J. Computat. Phys. 82, 64 (1989).

[20] J. Quirk, Ph.D. Thesis, Cranfield Institute of Technology (1991).

[21] D. DeZeeuw and K. Powell, J. Computat. Phys. 104, 56 (1993).

[22] L. Wild and B. Schutz (in preparation).

[23] P. Anninos, J. Masso, E. Seidel, W. Suen, and M. Tobias, Phys. Rev. D 56, 842 (1997).

[24] B. Szilágyi, R. Gómez, N. Bishop, and J. Winicour, grqc/9912030.

[25] H. O. Kreiss and J. Oliger, Methods for the Approximate Solution of Time Dependent Problems, GARP Publication Series No. 10 (World Meteorological Organization, Geneva, 1973).

[26] W. Landry and S. A. Teukolsky, gr-qc/9912004. 


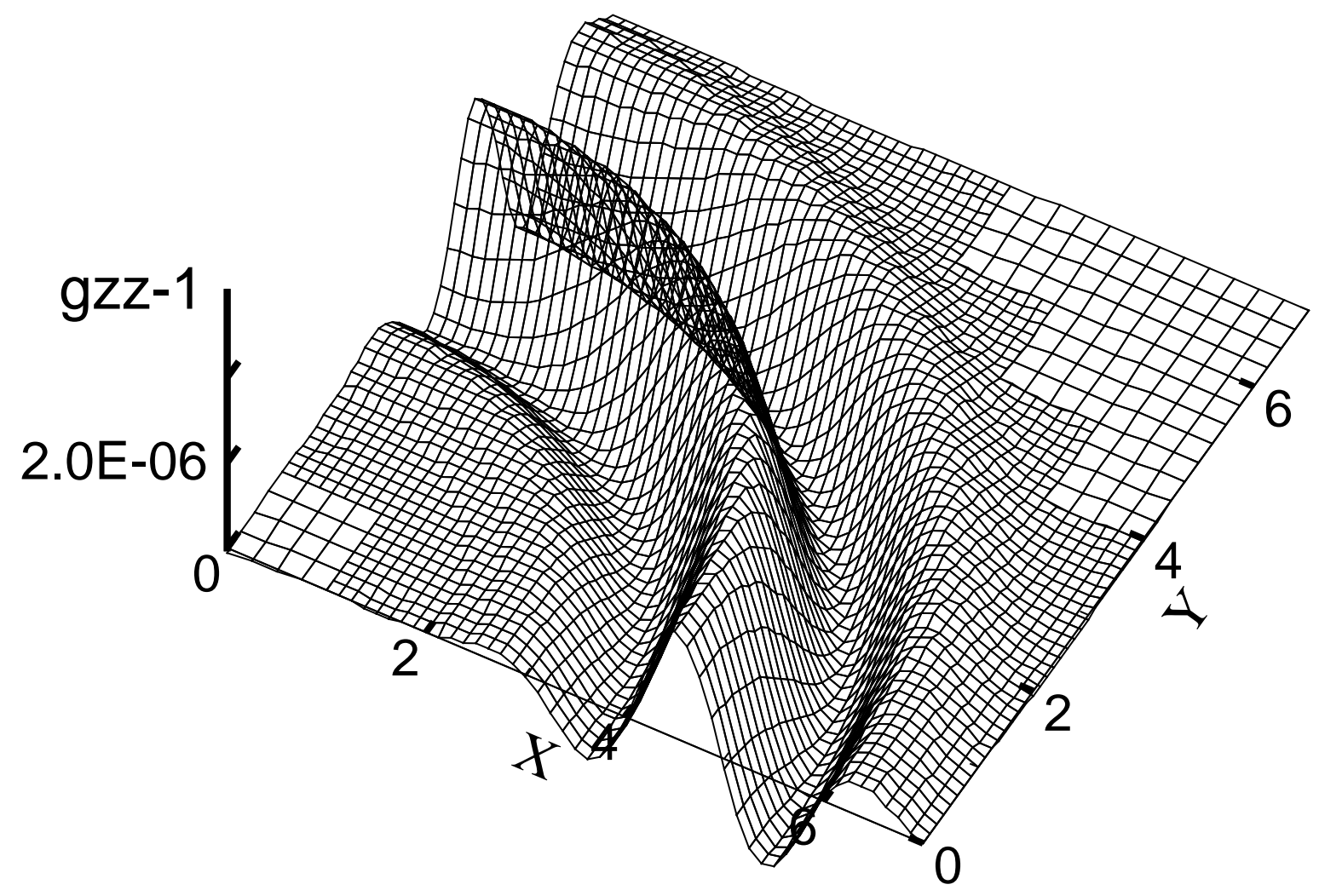

FIG. 1. The metric component $g_{z z}$ is shown in the equato-

rial plane at time $t=5.06$ in the 2-level AMR run. 


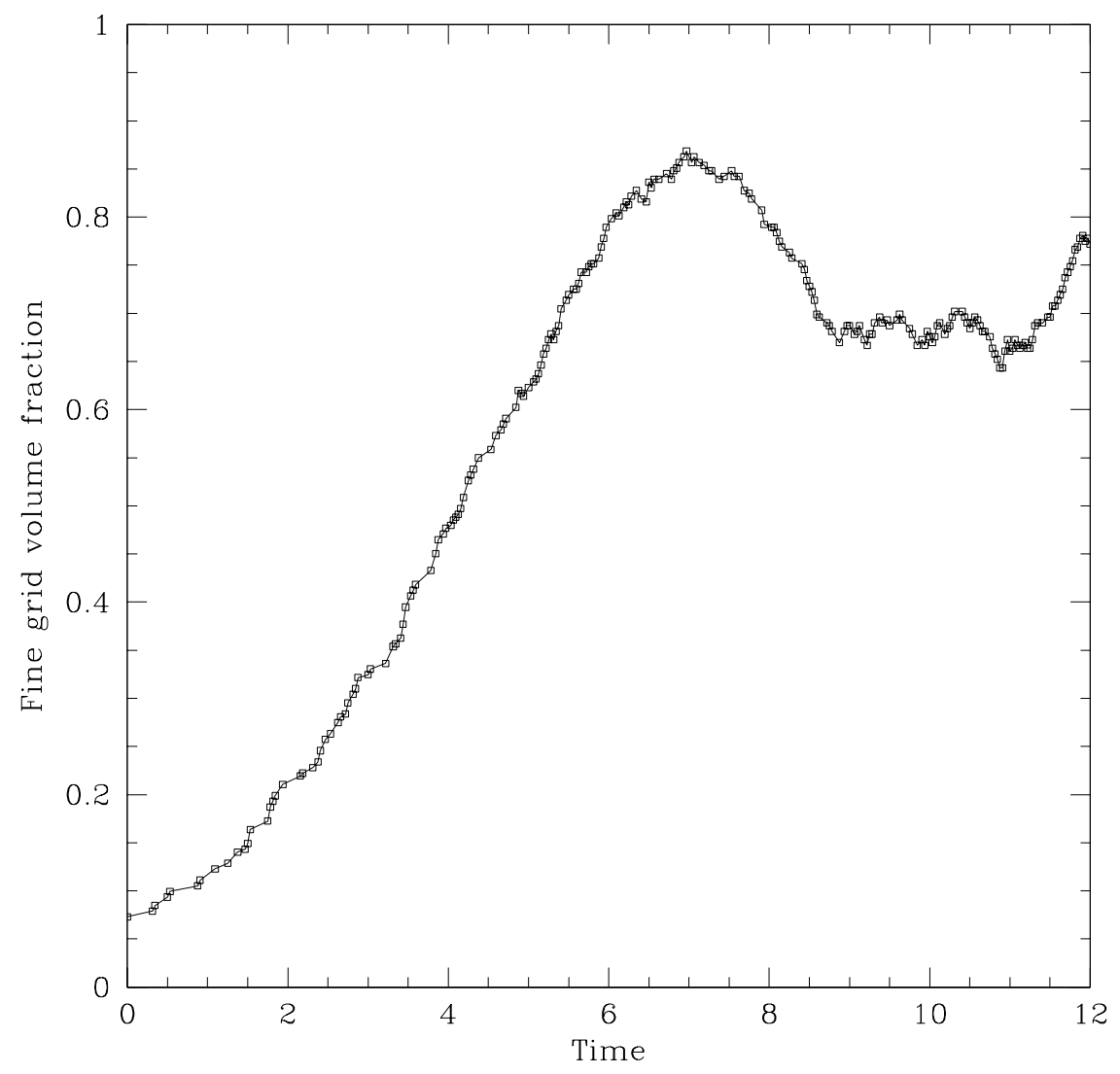

FIG. 3. The fraction of the computational volume covered by the fine grid is shown versus time for the AMR run in Fig. 1 